\title{
Effective solution of two simultaneous Pell equations by the elliptic logarithm method
}

\author{
by \\ N. Tzanakis (Iraklion)
}

To my wife Maro on the occasion of the silver jubilee of our common life

1. Introduction. The purpose of this paper is to present a hopefully practical and uniform general method $\left({ }^{1}\right)$ for solving explicitly any specific system of two Pell equations.

Clearly, any such system can be reduced to one of the form

$$
X^{2}-D_{1} Y^{2}=A_{1}, \quad Z^{2}-D_{2} Y^{2}=A_{2} \quad \text { in positive integers } X, Y, Z,
$$

where $A_{1}, A_{2}$ are non-zero integers, $D_{1}, D_{2}$ are non-square positive integers and $A_{1} D_{2}-A_{2} D_{1} \neq 0$. Note that, if the last condition is not satisfied, then $A_{2} / A_{1}=D_{2} / D_{1}=q^{2}$ for some $q \in \mathbb{Q}$ and the solutions $(X, Y, Z)$ of the system of equations are given by $(X, Y, q X)$, where $(X, Y)$ is a solution of the first equation of the system.

A large number of papers are devoted to the study of simultaneous Pell equations $\left({ }^{2}\right)$; they could be classified into three categories:

The first one includes papers in which results are proved of the type "there are no solutions", or "there are at most $k$ solutions", where $k$ is a number between, say, 1 and 4; such are the papers [4]-[8], [20], [21], [26], [38]-[40]. The generality of such results is obtained at the cost of restricting at least two parameters among $A_{1}, D_{1}, A_{2}, D_{2}$. Of course, if a system (1) falls within the scope of such a paper and $\left(^{3}\right)$ one is so lucky that the number of solutions he actually knows is equal to the maximum number of solutions proved in the paper, the system is completely solved.

2000 Mathematics Subject Classification: 11D09, 11G05, 11 Y50.

( $\left.{ }^{1}\right)$ Throughout this paper the term "algorithm", in a broad sense though, could also be used in place of the word "method".

$\left({ }^{2}\right)$ Often, solving (1) is reduced to finding common terms of two distinct second order recurrence sequences, a problem studied in a number of papers without any direct reference to (1); in our bibliography it is natural to include such papers.

$\left({ }^{3}\right)$ This is the very optimistic case! 
In a second category we put papers dealing with the explicit solution of some system(s), in which each parameter $A_{1}, D_{1}, A_{2}, D_{2}$ has either a specific numerical value or a certain special form. In these papers, either Baker's theory is applied or, quite often, elegant elementary arguments; see [2], [3], [9], [13]-[16], [18], [24], [25], [28], [37].

A third category includes papers in which effective but not explicit results are proved; see [19], [22], [23].

From the content and the results of the above papers it is clear that their objects are distinct from that of the present paper $\left({ }^{4}\right)$. To the best of the author's knowledge, only two works in the literature deal with a similar object $\left({ }^{5}\right)$ :

First, W. S. Anglin's book [1], Section 4.6, where a method is proposed for solving (1) when all parameters are absolutely less than 1000. In principle, that method, which is based on the Theory of Linear Forms in Complex Logarithms, can work in general, even without the above restriction on the size of the parameters. Its complexity, however, depends upon the number of prime factors of $A_{1}$ and $A_{2}$, while the method proposed in the present paper, based on the Theory of Linear Forms in Elliptic Logarithms, might be free of this defect $\left({ }^{6}\right)$. Nice examples with a vivid discussion on the comparison of the Complex Logarithm versus the Elliptic Logarithm method are found in [34].

Second, the paper [27] by R. G. E. Pinch, where the solution of (1) is reduced to a finite number of problems of the form: "Given two distinct second order recurrence sequences $\left(X_{k}\right)$ and $\left(Y_{l}\right)$ assuming integer values and an integer $g$, show that $(0,0)$ is the only pair $(n, m)$ satisfying $X_{n}=$ $Y_{m}+g(*)$ ". A first step of the method consists in obtaining an upper bound $B$ of $n$, using again the Theory of Linear Forms in Complex Logarithms. Then an algorithm builds up, step by step, sets $P$ and $Q$ of primes with certain properties. The main property of the set $P$ is that its elements are the prime factors of an integer $N$ such that, if $(*)$ is satisfied, then $n \equiv 0$ $(\bmod N)$. One expects to build $P$ so large that $N>B$, which will force $n=0$. It is not known a priori how large the primes in the sets $P, Q$ have to be $\left({ }^{7}\right)$; on the other hand, there is no theoretical guarantee that, in any specific case, the method will work with sets $P, Q$ including primes of "reasonable" size.

$\left({ }^{4}\right)$ Cf. the very beginning of our Introduction.

$\left({ }^{5}\right)$ We do not include among them paper [24] in which an elementary interesting method is proposed which, however, could not be considered "general".

$\left({ }^{6}\right)$ I owe this remark to the referee.

$\left({ }^{7}\right)$ In [35], where we had to solve the system $U^{2}-5 V^{2}=-4 \mu, U^{2}-10 Z^{2}=\mu, \mu= \pm 1$, we applied Pinch's method; the sets $P$ and $Q$ turned out to include the 40 primes from 2 to 173 and the 145 primes from 3 to 997 , respectively. 
In the present paper, we adopt a quite different approach, using $E l$ liptic Curves and Linear Forms of Elliptic Logarithms. The method that we propose depends upon the theoretically non-effective computation of a Mordell-Weil basis for a certain elliptic curve. This, in practice, causes no real problems, given the current state of the art on the actual computation of Mordell-Weil bases and the large positive experience from the extended application of the elliptic curves techniques to the explicit solution of diophantine equations. A more serious problem from the application of the method can possibly arise when the rank of the related elliptic curve is high, say, larger than 7 or 8 . In such a case $\left(^{8}\right)$, the final and theoretically easiest step, which we describe after relation (21), may turn out a difficult computational task.

Finally, we wish to stress the following fact: As the reader will realize, for the application of our method we assume the knowledge of a non-trivial rational solution $\left(X_{0}, Y_{0}, Z_{0}\right)$. What if we can neither detect such a solution nor prove its non-existence by, say, congruence considerations? Note that there are very efficient methods for deciding whether each equation in (1), considered separately, has a rational solution; see, for example, [11] and the references therein. Thus, if we know that both equations have rational solutions, but we are unable to decide whether the system of the two equations has a rational solution $\left({ }^{9}\right)$, we can work, at least in principle, as follows: We effectively obtain a rational solution $\left(X_{0}, Y_{0}\right)$, say, of the first equation, so that $\left(X_{0}, Y_{0}, Z_{0}\right), Z_{0}=\sqrt{A_{2}+D_{2} Y_{0}^{2}}$ is a solution belonging to at most a quadratic extension $\mathbb{K}$ of $\mathbb{Q}$ and the method of this paper remains exactly the same with $\mathbb{K}$ in place of $\mathbb{Q}$. In practice, however, this implies working with elliptic curves over $\mathbb{K}$ and the state of the art of this subject, from the computational point of view, is not satisfactory today. Thus, although the method developed in the present paper still works in this case, we cannot, at present, be very optimistic about the happy outcome of the computations related to the relevant elliptic curve.

Acknowledgements. I am grateful to my colleague J. Antoniadis and the referee, who drew my attention to [1], an important reference which I had missed out in the first version of the paper. I also thank Nils Bruin for his very challenging electronic comments.

2. The method. We assume that a rational solution $\left(X_{0}, Y_{0}, Z_{0}\right)$ is known with $Y_{0}>0$ and $X_{0}, Z_{0} \geq 0$, not both zero.

$\left({ }^{8}\right)$ Very unlikely to happen, we believe, if the parameters in (1) are not constructed on purpose.

$\left({ }^{9}\right)$ It is worth asking whether such a case can occur in practice. 
2.1. The related elliptic curve and the birational transformation. Elimination of $A_{1}, A_{2}$ from the two equations gives

$$
A_{2} X^{2}-A_{1} Z^{2}+\left(A_{1} D_{2}-A_{2} D_{1}\right) Y^{2}=0 .
$$

We put

$$
u=\frac{Y_{0} X-X_{0} Y}{Y_{0} Z-Z_{0} Y}
$$

so that

$$
Y=\frac{Y_{0}(X-u Z)}{X_{0}-u Z_{0}}
$$

and substitute in (2) to obtain a quadratic equation in $X / Z$. Obviously, $X / Z=X_{0} / Z_{0}$ is one root of this quadratic equation, whence it follows that the second root is

$$
\frac{X}{Z}=\frac{-A_{2} X_{0} u^{2}+2 A_{1} Z_{0} u-A_{1} X_{0}}{A_{2} Z_{0} u^{2}-2 A_{2} X_{0} u+A_{1} Z_{0}} .
$$

Then, by the expression of $Y$ in terms of $X, Z$ and $u$, above, we obtain

$$
\frac{Y}{Z}=\frac{\left(-A_{2} u^{2}+A_{1}\right) Y_{0}}{-A_{2} Z_{0} u^{2}+2 A_{2} X_{0} u-A_{1} Z_{0}} .
$$

We have $1-D_{2}(Y / Z)^{2}=A_{2} / Z^{2}$, hence, by (5) and the relations

$$
X_{0}^{2}-D_{1} Y_{0}^{2}=A_{1}, \quad Z_{0}^{2}-D_{2} Y_{0}^{2}=A_{2},
$$

it follows that

$$
a u^{4}+b u^{3}+c u^{2}+d u+e^{2}=v^{2},
$$

where

$$
v=\sigma \frac{-A_{2} Z_{0} u^{2}+2 A_{2} X_{0} u-A_{1} Z_{0}}{Z}, \quad \sigma=\operatorname{sgn}\left(A_{1}\right)
$$

and

$$
\begin{gathered}
a=A_{2}^{2}, \quad b=-4 A_{2} X_{0} Z_{0}, \quad c=4 A_{1} Z_{0}^{2}-2 A_{1} A_{2}+4 A_{2} X_{0}^{2}, \\
d=-4 A_{1} X_{0} Z_{0}, \quad e=\left|A_{1}\right| .
\end{gathered}
$$

Later we will justify the choice of $\sigma$ in (8). Using (3) in (8) and taking into account (6) we express $v$ as a rational function of $X, Y, Z$ :

$$
v=2 \sigma Y_{0}^{2} \frac{A_{2} X_{0} X+\left(D_{2} A_{1}-D_{1} A_{2}\right) Y_{0} Y-A_{1} Z_{0} Z}{\left(Y_{0} Z-Z_{0} Y\right)^{2}} .
$$

Conversely, we need also express $X, Y, Z$ as rational functions of $u, v$. By the definition of $v$,

$$
Z=\sigma \frac{-A_{2} Z_{0} u^{2}+2 A_{2} X_{0} u-A_{1} Z_{0}}{v}
$$


and this, combined with (5), (4) and (6) gives

$$
Y=\sigma \frac{\left(-A_{2} u^{2}+A_{1}\right) Y_{0}}{v}, \quad X=\sigma \frac{A_{2} X_{0} u^{2}-2 A_{1} Z_{0} u+A_{1} X_{0}}{v} .
$$

We consider now (7). Following Section 2 of [36], we define the rational functions

$$
\begin{aligned}
\mathbf{x}(u, v)= & \frac{2 e v+d u+2 e^{2}}{u^{2}}+\frac{c}{3} \\
\sigma \cdot \mathbf{y}(u, v)= & \frac{8 e^{3} v+8 e^{4}+4 e^{2} d u+\left(4 e^{2} c-d^{2}\right) u^{2}}{2 e u^{3}} \\
& +\frac{d}{2 e} \mathbf{x}(u, v)-\frac{c d}{6 e}+b e \\
\mathbf{u}(x, y)= & \frac{12 e^{2} x+8 e^{2} c-3 d^{2}}{2\left(6 \sigma y e-3 d x+c d-6 b e^{2}\right)} \\
\mathbf{v}(x, y)= & \frac{3 x-c}{6 e} \mathbf{u}(x, y)^{2}-\frac{d}{2 e} \mathbf{u}(x, y)-e
\end{aligned}
$$

where, as before, $\sigma=\operatorname{sgn}\left(A_{1}\right)$. The functions

$$
\begin{aligned}
& (u, v) \mapsto(x, y)=(\mathbf{x}(u, v), \mathbf{y}(u, v)), \\
& (x, y) \mapsto(u, v)=(\mathbf{u}(x, y), \mathbf{v}(x, y))
\end{aligned}
$$

establish a birational transformation between (7) and

$$
y^{2}=x^{3}+A x+B,
$$

where

$$
A=-\frac{1}{3} c^{2}+b d-4 a e^{2}, \quad B=\frac{2}{27} c^{3}-\frac{1}{3} b c d-\frac{8}{3} a c e^{2}+b^{2} e^{2}+a d^{2} .
$$

So far, our discussion sums up to the following:

Proposition 2.1. Let $(X, Y, Z)$ be a solution of the initial system (1). Using (3) and (10) we obtain $(u, v)$ satisfying (7) and then, by (17), we obtain $(x, y)$ satisfying (19). This gives a birational transformation from (1) to (19), say $x=\mathcal{X}(X, Y, Z), y=\mathcal{Y}(X, Y, Z)$. Conversely, if $(x, y)$ satisfies (19), then, using (18), we obtain $(u, v)$ satisfying (7) and then, by (11) and (12), we obtain a solution $(X, Y, Z)$ of (1).

2.2. Some technical results. The following result is useful for the practical application of our method.

Proposition 2.2. The right-hand side of (19) has three distinct rational roots, namely

$$
\begin{aligned}
& \varrho_{1}=\frac{4}{3}\left(D_{2} X_{0}^{2}-2 D_{1} D_{2} Y_{0}^{2}+D_{1} Z_{0}^{2}\right) Y_{0}^{2}=\frac{4}{3}\left(A_{1} D_{2}+A_{2} D_{1}\right) Y_{0}^{2}, \\
& \varrho_{2}=\frac{4}{3}\left(-2 D_{2} X_{0}^{2}+D_{1} D_{2} Y_{0}^{2}+D_{1} Z_{0}^{2}\right) Y_{0}^{2}=\frac{4}{3}\left(A_{2} D_{1}-2 A_{1} D_{2}\right) Y_{0}^{2}, \\
& \varrho_{3}=\frac{4}{3}\left(D_{2} X_{0}^{2}+D_{1} D_{2} Y_{0}^{2}-2 D_{1} Z_{0}^{2}\right) Y_{0}^{2}=\frac{4}{3}\left(A_{1} D_{2}-2 A_{2} D_{1}\right) Y_{0}^{2},
\end{aligned}
$$


hence, the curve $\mathcal{E}$ defined by (19) is an elliptic curve. Denote by $e_{1}, e_{2}, e_{3}$, respectively, the largest, middle and least among the three roots above. Then the following table gives the values of $e_{1}, e_{2}, e_{3}$ :

\begin{tabular}{ccc|ccc}
\hline$A_{1}$ & $A_{2}$ & $A_{2} D_{1}-A_{1} D_{2}$ & $e_{3}$ & $e_{2}$ & $e_{1}$ \\
\hline$>0$ & $>0$ & $>0$ & $\varrho_{3}$ & $\varrho_{2}$ & $\varrho_{1}$ \\
$>0$ & $>0$ & $<0$ & $\varrho_{2}$ & $\varrho_{3}$ & $\varrho_{1}$ \\
$>0$ & $<0$ & any sign & $\varrho_{2}$ & $\varrho_{1}$ & $\varrho_{3}$ \\
$<0$ & $>0$ & any sign & $\varrho_{3}$ & $\varrho_{1}$ & $\varrho_{2}$ \\
$<0$ & $<0$ & $>0$ & $\varrho_{1}$ & $\varrho_{3}$ & $\varrho_{2}$ \\
$<0$ & $<0$ & $<0$ & $\varrho_{1}$ & $\varrho_{2}$ & $\varrho_{3}$ \\
\hline
\end{tabular}

Moreover, define

$$
\begin{aligned}
& x_{0}=\lim _{Y \rightarrow+\infty} \mathcal{X}\left(\sqrt{A_{1}+D_{1} Y^{2}}, Y,-\sqrt{A_{2}+D_{2} Y^{2}}\right), \\
& y_{0}=\lim _{Y \rightarrow+\infty} \mathcal{Y}\left(\sqrt{A_{1}+D_{1} Y^{2}}, Y,-\sqrt{A_{2}+D_{2} Y^{2}}\right) .
\end{aligned}
$$

Then $x_{0}, y_{0} \in \mathbb{Q}\left(\sqrt{D_{1}}, \sqrt{D_{2}}\right), x_{0}>e_{1}, y_{0}>0$ and $\left(x_{0}, y_{0}\right)$ is a point on $\mathcal{E}_{0}$, the zero-component of the elliptic curve $\mathcal{E}$.

Finally, for any positive solution $(X, Y, Z)$ of (1), with $Y$ sufficiently large, the point $(x, y)=(\mathcal{X}(X, Y,-Z), \mathcal{Y}(X, Y,-Z))$ belongs to $\mathcal{E}_{0}$ and $y>0$; how large $Y$ must be, can be made explicit in any particular case (1).

Proof. With Maple V we easily calculated the three roots. These are distinct, in view of our initial restrictions on the parameters of (1). Every claim in the table can be checked without much difficulty, but this is probably a rather tedious task without the aid of a Computer Algebra package. The same is true for the computation of $x_{0}$ and $y_{0}$, the values of which are

$$
\begin{aligned}
& x_{0}= \frac{4 Y_{0}^{2}}{3}\left(D_{2} X_{0}^{2}+D_{1} D_{2} Y_{0}^{2}+D_{1} Z_{0}^{2}+3 \sqrt{D_{1}} D_{2} X_{0} Y_{0}\right. \\
&\left.+3 D_{1} \sqrt{D_{2}} Y_{0} Z_{0}+3 \sqrt{D_{1} D_{2}} X_{0} Z_{0}\right), \\
& y_{0}=\frac{8 \sigma\left|A_{1}\right| Y_{0}^{3} \sqrt{D_{1}}\left(Z_{0} \sqrt{D_{1}}+X_{0} \sqrt{D_{2}}\right)\left(Z_{0}+Y_{0} \sqrt{D_{2}}\right)}{X_{0}-Y_{0} \sqrt{D_{1}}} .
\end{aligned}
$$

It is now clear that $x_{0}$ is positive and larger than all three roots $\varrho_{1}, \varrho_{2}, \varrho_{3}$. On the other hand, $\operatorname{sgn}\left(X_{0}-Y_{0} \sqrt{D_{1}}\right)=\operatorname{sgn}\left(A_{1}\right)=\sigma$, hence $y_{0}>0$. Finally, if $(X, Y, Z)$ is a positive solution of (1), then $X=\sqrt{A_{1}+D_{1} Y^{2}}, Z=$ $\sqrt{A_{2}+D_{2} Y^{2}}$, hence, for sufficiently large $Y$, the number $\mathcal{X}(X, Y,-Z)$ is sufficiently close to $x_{0}$, which in turn is larger than $e_{1}$; this, obviously, proves that $(x, y) \in \mathcal{E}_{0}$ and $y>0$ in view of the fact that, for sufficiently large $Y$, $y$ is near $y_{0}$. 
We also need the following

Lemma 2.3. The substitution $\left(\sqrt{A_{1}+D_{1} Y^{2}},-\sqrt{A_{2}+D_{2} Y^{2}}\right) \leftarrow(X, Z)$ in (10) results in a function $v=v(Y)$, which, for sufficiently large $Y$, assumes positive values.

Proof. In (10), the factor that determines the sign of $v$ is (after the substitution mentioned in the lemma)

$$
\sigma\left(A_{1} Z_{0} \sqrt{A_{2}+D_{2} Y^{2}}+\left(D_{2} A_{1}-D_{1} A_{2}\right) Y_{0} Y+A_{2} X_{0} \sqrt{A_{1}+D_{1} Y^{2}}\right) .
$$

Dividing this by $Y$ and letting $Y \rightarrow+\infty$, we find the limit

$$
\sigma\left(X_{0}-Y_{0} \sqrt{D_{1}}\right)\left(Z_{0} \sqrt{D_{1}}+X_{0} \sqrt{D_{2}}\right)\left(Z_{0}+Y_{0} \sqrt{D_{2}}\right)
$$

which is positive, as we saw in the proof of Proposition 2.2. This, certainly, implies that, for sufficiently large $Y$, the corresponding $v$ is positive.

The following result is implicitly needed for our method.

LEMMA 2.4. There exists a positive constant $\Psi_{0}$, explicitly computable in every particular instance of the system (1), such that the bijection

$$
\begin{aligned}
{\left[\Psi_{0},+\infty\right) \ni Y \rightarrow \mathcal{X}\left(\sqrt{A_{1}+D_{1} Y^{2}}, Y,-\sqrt{A_{2}+D_{2} Y^{2}}\right) } \\
\quad \in\left[\mathcal{X}\left(\sqrt{A_{1}+D_{1} \Psi_{0}^{2}}, \Psi_{0},-\sqrt{A_{2}+D_{2} \Psi_{0}^{2}}\right), x_{0}\right)
\end{aligned}
$$

is strictly decreasing.

Proof. The proof is elementary, but quite tedious; once again we turned to the aid of MAPLE $V$. The function $\mathcal{X}\left(\sqrt{A_{1}+D_{1} Y^{2}}, Y,-\sqrt{A_{2}+D_{2} Y^{2}}\right)$ is the composition $h_{2} \circ h_{1}$ of the functions

$$
\begin{array}{lll}
h_{1}: & Y \mapsto u=\frac{X_{0} Y-Y_{0} \sqrt{A_{1}+D_{1} Y^{2}}}{Y_{0} \sqrt{A_{2}+D_{2} Y^{2}}+Z_{0} Y} \quad(\text { cf. (3)), } \\
h_{2}: & u \mapsto \mathbf{x}\left(u, \sqrt{a u^{4}+b u^{3}+c u^{2}+d u+e^{2}}\right) \quad \text { (cf. (13) and (10)), }
\end{array}
$$

with $a, b, c, d, e$ as defined in (9). A small explanation on the definition of $h_{2}$ : The arguments of the function $\mathbf{x}$ in (13) are $u=h_{1}(Y)$ and the $v$ obtained from $(10)$ after the substitution $(X, Z) \leftarrow\left(\sqrt{A_{1}+D_{1} Y^{2}},-\sqrt{A_{2}+D_{2} Y^{2}}\right)$. By Lemma 2.3, this value of $v$ is positive, for sufficiently large $Y$, hence, by (7), it is expressed as $\sqrt{a u^{4}+b u^{3}+c u^{2}+d u+e^{2}}\left({ }^{10}\right)$. A computation shows that (primed letters denote derivatives)

$$
\lim _{Y \rightarrow+\infty} h_{1}^{\prime}(Y)=\frac{Y_{0}\left(X_{0}-Y_{0} \sqrt{D_{1}}\right)\left(Z_{0} \sqrt{D_{1}}+X_{0} \sqrt{D_{2}}\right)}{\sqrt{D_{1} D_{2}}\left(Z_{0}+Y_{0} \sqrt{D_{2}}\right)} .
$$

Since $\operatorname{sgn}\left(X_{0}-Y_{0} \sqrt{D_{1}}\right)=\operatorname{sgn}\left(A_{1}\right)=\sigma$, this shows that, for sufficiently large $Y$, the function $h_{1}$ is strictly increasing if $\sigma=+1$ and strictly decreasing if

$\left({ }^{10}\right)$ We always use the symbol $\sqrt{ }$ to denote the positive square root. 
$\sigma=-1$. We also note that

$$
\lim _{Y \rightarrow+\infty} h_{1}(Y)=\frac{X_{0}-Y_{0} \sqrt{D_{1}}}{Z_{0}+Y_{0} \sqrt{D_{2}}}=: u_{0}
$$

Next, we consider the function $h_{2}(u)$ for $u$ sufficiently near to $u_{0}$. We write $h_{2}(u)=2 e \sqrt{h_{3}(u)}+h_{4}(u)$, where

$$
h_{3}(u)=a+\frac{b}{u}+\frac{c}{u^{2}}+\frac{d}{u^{3}}+\frac{e^{2}}{u^{4}}, \quad h_{4}(u)=\frac{d}{u}+\frac{2 e^{2}}{u^{2}}+\frac{c}{3} .
$$

We compute

$$
\begin{aligned}
h_{3}^{\prime}\left(u_{0}\right)= & -\frac{4 Y_{0}}{A_{1}}\left(X_{0}+Y_{0} \sqrt{D_{1} D_{2}}+Z_{0} \sqrt{D_{1}}\right) \\
& \times\left(X_{0}+Y_{0} \sqrt{D_{1}}\right)^{2}\left(Z_{0}+Y_{0} \sqrt{D_{2}}\right)^{2} \\
h_{4}^{\prime}\left(u_{0}\right)= & -\frac{8 Y_{0}^{3}}{A_{1}^{3}}\left(Z_{0} \sqrt{D_{1}}+X_{0} \sqrt{D_{2}}\right)\left(X_{0}+Y_{0} \sqrt{D_{1}}\right)^{3} \\
& \times\left(Z_{0}+Y_{0} \sqrt{D_{2}}\right)^{3} \sqrt{D_{1} D_{2}} .
\end{aligned}
$$

Thus, $\operatorname{sgn}\left(h_{3}^{\prime}\left(u_{0}\right)\right)=\operatorname{sgn}\left(h_{4}^{\prime}\left(u_{0}\right)\right)=-\sigma$, which shows that, near $u_{0}, h_{2}(u)$ is strictly decreasing if $\sigma=+1$ and strictly increasing if $\sigma=-1$. If we combine with the analogous conclusion for $h_{1}(u)$, we see that, for sufficiently large $Y$, the function $h_{2} \circ h_{1}(Y)$ is strictly decreasing, as claimed.

2.3. Elliptic integrals and linear forms in elliptic logarithms. Differentiating (3) and taking into account that $X d X=D_{1} d Y$ and $Z d X=D_{2} d Y$ we get

$$
d u=-\frac{Y_{0}\left(-A_{2} D_{1} Y_{0} Y+A_{2} X_{0} X+A_{1} D_{2} Y_{0} Y-A_{1} Z_{0} Z\right)}{X Z\left(Y_{0} Z-Z_{0} Y\right)^{2}} d Y .
$$

Then, taking into account (10) and the relation

$$
\frac{d x}{y}=-\sigma \frac{d u}{v}
$$

(see e.g. relation (2) of [33] $\left({ }^{11}\right)$ ), we conclude that

$$
\frac{d x}{y}=\frac{1}{2 Y_{0}} \cdot \frac{d Y}{X Z}
$$

From now on we will assume that $(X, Y, Z)$ is a positive integer solution of (1) with $Y$ sufficiently large (cf. Proposition 2.2 and Lemmas 2.3 and 2.4) and we will consider $P=(x(P), y(P))=(\mathcal{X}(X, Y,-Z), \mathcal{Y}(X, Y,-Z))$, which, by Proposition 2.2 , belongs to $\mathcal{E}_{0}(\mathbb{Q})$ and has $y(P)>0$. If necessary, we may also write $X(P), Y(P), Z(P)$ instead of $X, Y,-Z$. Conversely, by

$\left({ }^{11}\right)$ In the notation of that paper, $f(u, v)=a u^{4}+b u^{3}+c u^{2}+d u+e^{2}-v^{2}$ and a (symbolic) computation shows that $G(u, v)=-\sigma$. 
Proposition 2.1 , any point $P \in \mathcal{E}(\mathbb{Q})\left({ }^{12}\right)$ corresponds to a rational solution $(X(P), Y(P), Z(P))$ of $(1)$.

Assume now that we know a basis $P_{1}, \ldots, P_{r}$ for the torsion-free part of the Mordell-Weil group $\left.\mathcal{E}(Q){ }^{13}\right)$. Observe that, for any $P_{i}$, if $P_{i} \notin \mathcal{E}_{0}(\mathbb{Q})$, then $P_{i}+\left(e_{2}, 0\right) \in \mathcal{E}_{0}(\mathbb{Q})$. Therefore,

We can always choose the points of our basis so that $P_{1}, \ldots, P_{r}$ belong to $\mathcal{E}_{0}(\mathbb{Q})$.

We put

$$
P=m_{1} P_{1}+\ldots+m_{r} P_{r}+T,
$$

where $T$ denotes a torsion point which, necessarily, belongs to $\mathcal{E}_{0}(\mathbb{Q})$ and the $m_{i}$ 's are unknown integers. If we manage to bound

$$
M=\max \left\{\left|m_{1}\right|, \ldots,\left|m_{r}\right|\right\},
$$

then we can effectively solve, at least in principle, our system (1). Indeed, for any $\left(m_{1}, \ldots, m_{r}\right)$ satisfying this bound and any torsion point $T \in \mathcal{E}_{0}(\mathbb{Q})$, we compute the point $P=(x(P), y(P))$ and then, by the procedure described in Proposition 2.1, we obtain a rational solution $(X(P), Y(P), Z(P))$, which we accept if and only if all three $X(P), Y(P), Z(P)$ are integers.

A basic tool for our method is the usual isomorphism

$$
\phi: \mathcal{E}_{0}(\mathbb{R}) \rightarrow[0,1)=\mathbb{R} / \mathbb{Z}
$$

(see e.g. Section 2 of [31]). Let also $\omega=2 \int_{e_{1}}^{+\infty} d x / \sqrt{x^{3}+A x+B}$, the fundamental real period. From (21) we see that

$$
\phi(P)=m_{1} \phi\left(P_{1}\right)+\ldots+m_{r} \phi\left(P_{r}\right)+m_{0}+s / t
$$

where $m_{0} \in \mathbb{Z}$ and $s / t=\phi(T)$ with $0<s<t$ relatively prime integers, effectively bounded by a small number (see e.g. Section 4 of [31]). Moreover, since the $\phi$-values are positive and $<1$, it follows that

$$
\left|m_{0}\right| \leq r M \text {. }
$$

We now exhibit the close relation of $\phi(P)$ with an elliptic integral $\int \frac{d x}{y}$, where $y=\sqrt{x^{3}+A x+B}$. We will need the point $Q_{0}=\left(x_{0}, y_{0}\right) \in$ $\mathcal{E}_{0}\left(\sqrt{D_{1}}, \sqrt{D_{2}}\right)$, with $x_{0}, y_{0}$ as defined in Proposition 2.2. We have

$$
\int_{x(P)}^{x_{0}} \frac{d x}{y}=\int_{x(P)}^{+\infty} \frac{d x}{y}-\int_{x_{0}}^{+\infty} \frac{d x}{y}=\omega \phi(P)-\omega \phi\left(Q_{0}\right),
$$

by the definition of $\phi$. This, combined with (22) shows that the integral on the left-hand side of the above relation is equal to a linear form in elliptic

$\left({ }^{12}\right)$ Actually, we deal only with points $P \in \mathcal{E}_{0}(\mathbb{Q})$.

$\left({ }^{13}\right)$ This is, of course, the non-effective part of the method of this paper. 
logarithms:

$$
\int_{x(P)}^{x_{0}} \frac{d x}{y}=\mathcal{L}(P):=-\omega \phi\left(Q_{0}\right)+\left(m_{0}+\frac{s}{t}\right) \omega+m_{1} \omega \phi\left(P_{1}\right)+\ldots+m_{r} \omega \phi\left(P_{r}\right) .
$$

A symbolic computation using MAPLE $\mathrm{V}$ shows that

$$
2 \cdot Q_{0}=\left(\frac{4}{3}\left(D_{2} X_{0}^{2}+D_{1} D_{2} Y_{0}^{2}+D_{1} Z_{0}^{2}\right) Y_{0}^{2}, 8 D_{1} D_{2} X_{0} Y_{0}^{4} Z_{0}\right) .
$$

Therefore, $2 \cdot Q_{0} \in \mathcal{E}_{0}(\mathbb{Q})$, hence $2 \cdot Q_{0}=k_{1} P_{1}+\ldots+k_{r} P_{r}+T^{\prime}$, where $k_{1}, \ldots, k_{r}$ are explicitly known rational integers and $T^{\prime} \in \mathcal{E}_{0}(\mathbb{Q})$ is a torsion point. Then

$$
\phi\left(Q_{0}\right)=\frac{k_{1}}{2} \phi\left(P_{1}\right)+\ldots+\frac{k_{r}}{2} \phi\left(P_{r}\right)+\frac{k_{0}}{2}+\frac{s^{\prime}}{2 t^{\prime}},
$$

where $k_{0}$ is an explicitly known integer and $s^{\prime}, t^{\prime}$ have analogous properties to those of $s, t$ (see immediately after (22)). This transforms the expression of the linear form $\mathcal{L}(P)$ into

$$
\int_{x(P)}^{x_{0}} \frac{d x}{y}=\mathcal{L}(P)=\frac{p_{0}}{q_{0}} \omega+\frac{p_{1}}{2} \omega \phi\left(P_{1}\right)+\ldots+\frac{p_{r}}{2} \omega \phi\left(P_{r}\right),
$$

where $p_{0}, p_{1}, \ldots, p_{r}$ are rational (unknown) integers and $q_{0}$ is an explicitly known "small" integer $\left({ }^{14}\right)$. Moreover, it is clear that $\left({ }^{15}\right)$,

$$
N:=\max _{0 \leq i \leq r}\left|p_{i}\right| \leq \alpha M+\beta,
$$

where the "small" positive constants $\alpha, \beta$ can be explicitly calculated in every specific case.

2.4. Estimating the linear form in elliptic logarithms. The following steps lead now to an upper bound for $|\mathcal{L}(P)|$ in terms of $M$.

- By (20) and Lemma 2.4 we conclude $\left({ }^{16}\right)$

$$
\int_{x(P)}^{x_{0}} \frac{d x}{y}=-\frac{1}{2 Y_{0}} \int_{Y(P)}^{+\infty} \frac{d Y}{\sqrt{\left(A_{1}+D_{1} Y^{2}\right)\left(A_{2}+D_{2} Y^{2}\right)}},
$$

therefore, for sufficiently large $Y\left({ }^{17}\right),(24)$ and (26) imply that

$$
|\mathcal{L}(P)| \leq c_{1} Y^{-1}
$$

where $c_{1}$ is an explicit positive constant.

$\left({ }^{14}\right)$ Actually, $p_{0} / q_{0}=m_{0}-k_{0} / 2+s / t-s^{\prime} /\left(2 t^{\prime}\right)$ and $p_{i}=m_{i}-k_{i} / 2$ for $i=1, \ldots, r$.

$\left({ }^{15}\right)$ We use also (23) at this point.

$\left({ }^{16}\right)$ We always have in mind that $X=\sqrt{A_{1}+D_{1} Y^{2}}$ and $Z=-\sqrt{A_{2}+D_{2} Y^{2}}$.

$\left({ }^{17}\right)$ This is easily made explicit. 
- $x(P)$ is an explicit rational function $\mathcal{X}(X, Y, Z)$, hence, for the height $\mathrm{h}(x(P))$, we can find an explicit upper bound in terms of $X, Y, Z\left({ }^{18}\right)$. Then, since $X=\sqrt{A_{1}+D_{1} Y^{2}}, Z=-\sqrt{A_{2}+D_{2} Y^{2}}$, it follows that

$$
\mathrm{h}(x(P)) \leq c_{2}+2 \log Y,
$$

where $c_{2}$ is an explicit positive constant.

- We have an inequality of the form

$$
\widehat{\mathrm{h}}(P)-\frac{1}{2} \mathrm{~h}(x(P)) \leq c_{3},
$$

where $\widehat{\mathrm{h}}(\cdot)$ denotes the canonical height, as defined, for example, in Silverman's book [29], and $c_{3}$ is an explicit positive constant; see e.g. [30].

- We refer to (21). It is a well known fact that $\widehat{\mathrm{h}}(P)$ is a positive-definite quadratic form $q\left(m_{1}, \ldots, m_{r}\right)$, hence

$$
\widehat{\mathrm{h}}(P) \geq \lambda M^{2},
$$

where $\lambda$ is the least eigenvalue of the matrix of the quadratic form $q$, hence an explicit positive constant.

- An obvious combination of $(27)-(30)$ gives

$$
|\mathcal{L}(P)| \leq c_{1} \exp \left(0.5 c_{2}+c_{3}-\lambda M^{2}\right) .
$$

Next, an explicit lower bound for $|\mathcal{L}(P)|$ in terms of $N$ is obtained by a deep theorem due to S. David [12]. A version of this theorem adapted to our needs is found in the Appendix of [36]. According to Theorem 5 of that Appendix, either $N$ is "very small", or

$$
|\mathcal{L}(P)| \geq \exp \left(-c_{4}\left(\log N+c_{5}\right)\left(\log \log N+c_{6}\right)^{r+2}\right),
$$

where $c_{4}, c_{5}, c_{6}$ are explicit positive constants. Taking into account (25), we write the last relation as

$$
|\mathcal{L}(P)| \geq \exp \left(-c_{4}\left(\log (\alpha M+\beta)+c_{5}\right)\left(\log \log (\alpha M+\beta)+c_{6}\right)^{r+2}\right) .
$$

Since the lower bound in (32) is larger than the upper bound in (31) if $M$ is sufficiently large, it follows that $M$ must be bounded by some explicit upper bound. The upper bound for $M$ which is obtained in this way is huge. The rather large experimental experience accumulated so far shows that this bound is of the size of $10^{\left(5 r^{2}+15 r+28\right) / 2}$; see Section 3 of [32]. This bound is dramatically reduced with the use of the LLL Basis Reduction Algorithm. The reduction process has been extensively discussed in many relevant papers, so that there is no point to discuss it here once again; we refer the reader to Section 5 of [31], Section 5 of [36] and Section 6 of [17] $\left({ }^{19}\right)$.

$\left({ }^{18}\right)$ The fact that $X, Y, Z$ are integers is a crucial "detail" here.

$\left({ }^{19}\right)$ These are just three indicative references. 
3. Examples. In the examples of this section, all computations related to elliptic curves have been done by APECS of Ian Connell, a package based on MAPLE V. In all cases, the Mordell-Weil bases were computed unconditionally (no conjectures were assumed) and care was taken to transform the points of the basis to ones belonging to the zero-component of the curve, by adding the point $\left(e_{2}, 0\right)$ if necessary (cf. just before $(21)$ ).

Also, the results of Subsection 2.2 require that $Y$ be "sufficiently large". In every example, computations, very simple in practice, show that $Y \geq 10$ is more than enough.

3.1. The system with $D_{1}=5, A_{1}=19, D_{2}=3, A_{2}=-11$. A rational solution: $\left(X_{0}, Y_{0}, Z_{0}\right)=(8,3,4)$. The corresponding Weierstrass model is

$$
y^{2}=x^{3}-4064688 x+97538688 \text {. }
$$

The roots of the right-hand side are

$$
e_{3}=-2028, \quad e_{2}=24, \quad e_{1}=2004 .
$$

Relations (3) and (10) now become

$$
u=\frac{3 X-8 Y}{3 Z-4 Y}, \quad v=-72 \frac{22 X-84 Y+19 Z}{(3 Z-4 Y)^{2}},
$$

hence, by (13) and (17),

$$
\begin{aligned}
x=\frac{-12}{(3 X-8 Y)^{2}}\left(7477 Y^{2}-1\right. & 9152 Y-4008 X Y-3420 Y Z \\
& +1824 Z X+5016 X+4332 Z+11571),
\end{aligned}
$$

from which we find

$$
x_{0}=4884+2160 \sqrt{3}+2592 \sqrt{5}+1152 \sqrt{5} \sqrt{3} \approx 18882.7947568591741,
$$

and by (14) and (17),

$$
\begin{array}{r}
y=\frac{16416}{(3 X-8 Y)^{3}}\left(855 Y^{3}+7477 Y^{2}-456 X Y^{2}-2140 Z Y^{2}-4008 X Y-9519 Y\right. \\
+1002 X Z Y+456 Z Y+190 X Z+3344 X-1900 Z+17347) .
\end{array}
$$

Also, relations (11) and (12) give

$$
X=-8 \frac{11 u^{2}+19 u-19}{v}, \quad Y=3 \frac{11 u^{2}+19}{v}, \quad Z=\frac{44 u^{2}-176 u-76}{v},
$$

from which, by means of (15), (16) and (18), we obtain

$$
\begin{aligned}
& X=8 \frac{x^{3}+828 x^{2}+90 y x+15841008 x-20126660544+182520 y}{x^{3}-14652 x^{2}+4064688 x+19656858816-622080 y}, \\
& Y=3 \frac{x^{3}+4932 x^{2}+128 y x-12427344 x+20041387200-3072 y}{x^{3}-14652 x^{2}+4064688 x+19656858816-622080 y},
\end{aligned}
$$




$$
Z=-4 \frac{x^{3}+8892 x^{2}+216 y x-23737104 x-19181430720-432864 y}{x^{3}-14652 x^{2}+4064688 x+19656858816-622080 y} .
$$

A basis for the torsion-free part of $\mathcal{E}(\mathbb{Q})$, where $\mathcal{E}$ is the elliptic curve defined by $(33)$, is

$$
P_{1}=(7548,632016), \quad P_{2}=(112884,37920960)
$$

$\left(P_{1}, P_{2} \in \mathcal{E}_{0}(\mathbb{Q})\right)$ and $T=(2004,0)$ is the only torsion point on $\mathcal{E}_{0}(\mathbb{Q})$. With the aid of the function Expr of APECS we find that

$$
2 \cdot Q_{0}=(4884,311040)=P_{1}+P_{2} .
$$

We easily check that $\phi\left(2 \cdot Q_{0}\right)=\phi\left(P_{1}\right)+\phi\left(P_{2}\right)$ and $\phi(T)=1 / 2$. Therefore, if $P$ is a point on the elliptic curve which corresponds to an integer solution of our system of Pell equations and we put

$$
P=m_{1} P_{1}+m_{2} P_{2}+T, \quad M=\max \left\{\left|m_{1}\right|,\left|m_{2}\right|\right\},
$$

then the corresponding linear form in elliptic logarithms is

$$
\mathcal{L}(P)=\frac{p_{0}}{2} \omega+\frac{p_{1}}{2} \omega \phi\left(P_{1}\right)+\frac{p_{2}}{2} \omega \phi\left(P_{2}\right),
$$

where $p_{i}=2 m_{i}-1(i=1,2)$ and $p_{0}=2 m_{0}+s$ for some integers $m_{0}$ with $\left|m_{0}\right| \leq 2 M$ and $s \in\{0,1\}$ (cf. (23)). Therefore, in the notation of (25), $\alpha=4, \beta=1$. Also, $A_{1}+D_{1} Y^{2}=19+5 Y^{2}>5 Y^{2}$ and $A_{2}+D_{2} Y^{2}=$ $-11+3 Y^{2}>2.9725 Y^{2}$, provided that $Y>20$. Therefore, in (27) we can take $c_{1}=0.04324$. In the expression of $x$ as a rational function of $X, Y, Z$ we make the substitution $X=\sqrt{19+5 Y^{2}}, Z=-\sqrt{-11+3 Y^{2}}$; then it is straightforward to check that the maximum of the absolute values of the numerator and denominator is $\leq 376400 Y^{2}$, provided that $Y>20$; therefore in (28) we can take $c_{2}=\log 376400$. In (29) we use Silverman's result [30], which gives $c_{3}=5.84226214$. An easy program based on MAPLE $\mathrm{V}$ and APECS calculates the least eigenvalue of the positive-definite matrix related to $\widehat{\mathrm{h}}$, giving thus $\lambda=0.5179388$. Following the detailed steps described in the Appendix of [36] we calculate

$$
c_{4}=4.046622 \cdot 10^{74}, \quad c_{5}=2.570121, \quad c_{6}=30.018387
$$

and the comparison of the upper bound (31) with the lower bound (32) forces $M \leq 3.41 \cdot 10^{41}$. Following the reduction technique that uses the LLL basis reduction algorithm (as implemented in PARI), we can immediately reduce this huge upper bound down to 20 and then, by a second reduction step we reduce the bound to 7 . Finally, by a simple program that uses MAPLE and APECS we compute all combinations

$$
(x, y)=m_{1} P_{1}+m_{2} P_{2}+\varepsilon T, \quad-7 \leq m_{i} \leq 7(i=1,2), \varepsilon=0,1
$$

and for each we insert the values of $x$ and $y$ in the previously obtained formulas, which express $X, Y, Z$ as rational functions of $x, y$. The only positive integer triads $(X, Y, Z)$ obtained in this way are $(8,3,4)$ and $(12,5,8)$. Since 
the computation of certain $c_{i}$ 's has been done under the assumption $Y>20$, we also check which integer values in the range $1 \leq Y \leq 20$ are such that $\sqrt{19+5 Y^{2}}$ and $\sqrt{-11+3 Y^{2}}$ are both integers. No values of $Y$ besides 3 and 5 satisfy this condition, from which we conclude that the only positive integer solutions $(X, Y, Z)$ to the system (1) in this specific case are those two mentioned above.

3.2. The system with $D_{1}=7, A_{1}=2, D_{2}=32, A_{2}=-23$. This example is partially solved in [10], where it is proved that, if $(X, Y, Z)$ is a solution, then $X+Y \sqrt{7}<(3+\sqrt{7})(8+3 \sqrt{7})^{17^{192}}$. Here we give the complete solution. A rational solution: $\left(X_{0}, Y_{0}, Z_{0}\right)=(3,1,3)$. The corresponding Weierstrass model is

$$
y^{2}=x^{3}-\frac{645136}{3} x-\frac{692527232}{27}
$$

with right-hand side having roots

$$
e_{3}=-\frac{1156}{3}, \quad e_{2}=-\frac{388}{3}, \quad e_{1}=\frac{1544}{3} .
$$

Relations (3), (10) and (17) combined with (13) and (14), respectively, give

$$
\begin{array}{r}
x=\frac{8\left(-235-207 X+675 Y-18 Z+579 X Y+63 Y Z-27 Z X-1448 Y^{2}\right)}{3(X-3 Y)^{2}}, \\
y=\frac{-96}{(X-3 Y)^{3}}\left(-224 Y^{3}+96 X Y^{2}-1448 Y^{2}+504 Z Y^{2}+579 X Y-54 Z Y\right. \\
\quad+386 Y-193 X Z Y-138 X+63 Z-253+14 X Z),
\end{array}
$$

from the first of which we find

$$
x_{0}=\frac{2300}{3}+384 \sqrt{7}+336 \sqrt{2}+144 \sqrt{7} \sqrt{2} \approx 2796.609591 .
$$

Relations (11) and (12) combined with (15), (16) and (18), respectively, give

$$
\begin{aligned}
& X=3 \frac{27 x^{3}-108 x^{2}+756 y x+6552144 x+291312 y-3080761664}{27 x^{3}-62100 x^{2}+5806224 x-870912 y+5836492864}, \\
& Y=\frac{27 x^{3}+13716 x^{2}-11161008 x+972 y x+125712 y+2373881536}{27 x^{3}-62100 x^{2}+5806224 x-870912 y+5836492864}, \\
& Z=-3 \frac{27 x^{3}+48492 x^{2}+3456 y x-24422256 x-7899721664-1778688 y}{27 x^{3}-62100 x^{2}+5806224 x-870912 y+5836492864} .
\end{aligned}
$$

A basis for the torsion-free part of $\mathcal{E}(\mathbb{Q})$, where $\mathcal{E}$ is the elliptic curve defined by (34), consists of the single point $P_{1}=(8444 / 3,147200)$, and $T=(1544 / 3,0)$ is the only torsion point on $\mathcal{E}_{0}(\mathbb{Q})$. With the aid of the function Expr of APECS we find that $2 \cdot Q_{0}=(2300 / 3,16128)=-P_{1}+T$ and we easily check that $\phi\left(Q_{0}\right)=\frac{1}{4}-\frac{1}{2} \phi\left(P_{1}\right)$ and $\phi(T)=1 / 2$. If $P$ is a point on the elliptic curve, which corresponds to an integer solution of our system of Pell equations and we put $P=m_{1} P_{1}, M=\left|m_{1}\right|$, then the corresponding linear form in elliptic logarithms is 


$$
\mathcal{L}(P)=\frac{p_{0}}{2} \omega+\frac{p_{1}}{2} \omega \phi\left(P_{1}\right),
$$

where $p_{1}=2 m_{1}+1$ and $p_{0}=4 m_{0}+2 s-1$ for some integers $m_{0}$ with $\left|m_{0}\right| \leq M$ and $s \in\{0,1\}$ (cf. (23)). Therefore, in the notation of (25), $\alpha=4, \beta=1$.

Calculations completely analogous to those in Example 3.1 give $c_{1}=$ $0.03344, c_{2}=9.67, c_{3}=5.1074513, \lambda=0.69249, c_{4}=6.251 \cdot 10^{44}, c_{5}=2.752$, $c_{6}=34.56585$ and the comparison of the upper bound (31) and the lower bound (32) forces $M \leq 5.8 \cdot 10^{25}$. The reduction process gives the bound $M \leq 20$. We compute all combinations

$$
(x, y)=m_{1} P_{1}+\varepsilon T, \quad-20 \leq m_{1} \leq 20, \varepsilon=0,1,
$$

and for each we insert the values of $x$ and $y$ in the above expressions of $X, Y, Z$ as rational functions of $x, y$. The only positive integer triads $(X, Y, Z)$ obtained in this way are $(3,1,3)$ and $(717,271,1533)$. Since the computation of certain $c_{i}$ 's have been done under the assumption $Y>20$, we also check whether there are solutions $(X, Y, Z)$ with $1<Y \leq 20$, but this reveals no further solution. Therefore, the only positive integer solutions $(X, Y, Z)$ to the system (1) in this specific case are those two mentioned above.

3.3. Three examples taken from the literature. In this section we discuss, in brief, the application of our method to the solution of three examples taken from the literature.

The first is the well known example of A. Baker and H. Davenport [3]. Solutions are also given in [18] and [28]. In the notation of the present paper, $D_{1}=3, A_{1}=-2, D_{2}=8, A_{2}=-7$ and $\left(X_{0}, Y_{0}, Z_{0}\right)=(1,1,1)$. The parameters in the Weierstrass model (19) are $A=-5776 / 3, B=677248 / 27$. The rank of the curve is $1, P_{1}=(524 / 3,2240)$ being a basic point. Then the resolution of the system is completely analogous to that of Subsection 3.2 and proves that the only solutions in positive integers $(X, Y, Z)$ are $(1,1,1)$ and $(19,11,31)$.

A second example comes from the system $z^{2}-3 y^{2}=-2, z^{2}-6 x^{2}=-5$, studied in [37]. The substitution $(3 y, z, 6 x)=(X, Y, Z)$ gives a system (1) with $D_{1}=3, A_{1}=6, D_{2}=6, A_{2}=30$ and $\left(X_{0}, Y_{0}, Z_{0}\right)=(3,1,6)$. The parameters in the related Weierstrass elliptic curve (19) are $A=-32832$, $B=774144$. The rank of the curve is $1, P_{1}=(888,25920)$ being a basic point. As in the previous example, the resolution of the system is completely analogous to that of Subsection 3.2 and proves that the only solutions in positive integers $(X, Y, Z)$ are $(3,1,6)$ and $(123,71,174)$, hence the only positive integer solutions $(x, y, z)$ are $(1,1,1)$ and $(29,41,71)$.

The third example is found in [25] and corresponds to $D_{1}=5, A_{1}=$ $-20, D_{2}=2, A_{2}=1$ and $\left(X_{0}, Y_{0}, Z_{0}\right)=(0,2,3)$. The parameters in the related elliptic curve (19) are $A=-467200 / 3, B=-609280000 / 27$ and the 
rank of the curve is zero. The only rational point on the zero-component of the curve is the torsion point $T=(1360 / 3,0)$ and the correspondence $(x, y) \rightarrow(X, Y, Z)$ gives no further solution.

\section{References}

[1] W. S. Anglin, The Queen of Mathematics-An Introduction to Number Theory, Kluwer, Dordrecht, 1995.

[2] - Simultaneous Pell equations, Math. Comp. 65 (1996), 355-359.

[3] A. Baker and H. Davenport, The equations $3 x^{2}-2=y^{2}$ and $8 x^{2}-7=z^{2}$, Quart. J. Math. Oxford Ser. (2) 20 (1969), 129-137.

[4] M. A. Bennett, Solving families of simultaneous Pell equations, J. Number Theory 67 (1997), 246-251.

[5] - On the number of solutions to simultaneous Pell equations, J. Reine Angew. Math. 498 (1998), 173-199.

[6] -, On consecutive integers of the form $a x^{2}, b y^{2}$ and $c z^{2}$, Acta Arith. 88 (1999), 363-370.

[7] M. A. Bennett and P. G. Walsh, Simultaneous quadratic equations with few or no solutions, Indag. Math. (N.S.) 11 (2000), 1-12.

[8] J. H. Chen, Common solutions of the Pell equations $x^{2}-2 y^{2}=1$ and $y^{2}-D z^{2}=4$, J. Wuhan Univ. Natur. Sci. Ed. 1990, no. 1, 8-12 (in Chinese).

[9] Z. Y. Chen, The diophantine system of equations $5 x^{2}-3 y^{2}=2,16 y^{2}-5 z^{2}=11$, J. Central China Normal Univ. Natur. Sci. 30 (1996), 381-384 (in Chinese).

[10] _ , Upper bounds for positive integer solutions of the indeterminate equations $x^{2}-$ $7 y^{2}=2, z^{2}-32 y^{2}=-23$, ibid. 31 (1997), 253-256 (in Chinese).

[11] J. E. Cremona and D. Rusin, Efficient solution of rational conics, Math. Comp., to appear.

[12] S. David, Minorations de formes linéaires de logarithmes elliptiques, Mém. Soc. Math. France (N.S.) 62 (1995).

[13] A. Dujella, An absolute bound for the size of Diophantine $m$-tuples, J. Number Theory 89 (2001), 126-150.

[14] - Complete solution of a family of simultaneous Pellian equations, Acta Math. Inform. Univ. Ostraviensis 6 (1998), 59-67.

[15] A. Dujella and B. Jadrijević, A parametric family of quartic Thue equations, Acta Arith. 101 (2002), 159-170.

[16] A. Dujella and A. Pethő, A generalization of a theorem of Baker and Davenport, Quart. J. Math. Oxford Ser. (2) 49 (1998), 291-306.

[17] J. Gebel, A. Pethő and H. G. Zimmer, Computing integral points on elliptic curves, Acta Arith. 68 (1994), 171-192.

[18] P. Kanagasabapathy and T. Ponnudurai, The simultaneous diophantine equations $y^{2}-3 x^{2}=-2$ and $z^{2}-8 x^{2}=-7$, Quart. J. Math. Oxford Ser. (2) 26 (1975), 275-278.

[19] P. Kiss, On common terms of linear recurrences, Acta Math. Acad. Sci. Hungar. 40 (1982), 119-123.

[20] W. Ljunggren, A note on simultaneous Pell equations, Norsk. Mat. Tidsskr. 23 (1941), 132-138 (in Norwegian).

[21] D. W. Masser and J. H. Rickert, Simultaneous Pell equations, J. Number Theory 61 (1996), 52-66. 
[22] M. Mignotte, Intersection des images de certaines suites récurrentes linéaires, Theoret. Comput. Sci. 7 (1978), 117-122.

[23] —, Une extension du théorème de Skolem-Mahler, C. R. Acad. Sci. Paris Sér. A 288 (1979), 233-235.

[24] S. P. Mohanty and A. M. S. Ramasamy, The characteristic number of two simultaneous Pell's equations and its applications, Simon Stevin 59 (1985), 203-214.

[25] - - , The simultaneous diophantine equations $5 y^{2}-20=X^{2}$ and $2 y^{2}+1=Z^{2}$, J. Number Theory 18 (1984), 356-359.

[26] J. Pan, Y. Zhang and R. Zou, The Pell equations $x^{2}-8 y^{2}=1$ and $y^{2}-D z^{2}=1$, Chinese Quart. J. Math. 14 (1999), 73-77.

[27] R. G. E. Pinch, Simultaneous Pellian equations, Math. Proc. Cambridge Philos. Soc. 103 (1988), 35-46.

[28] G. Sansone, Il sistema diofanteo $N+1=x^{2}, 3 N+1=y^{2}, 8 N+1=z^{2}$, Ann. Mat. Pura Appl. 111 (1976), 125-151.

[29] J. H. Silverman, The Arithmetic of Elliptic Curves, Grad. Texts in Math. 106, Springer, Berlin, 1986.

[30] - , The difference between the Weil height and the canonical height on elliptic curves, Math. Comp. 55 (1990), 723-743.

[31] R. J. Stroeker and N. Tzanakis, Solving elliptic diophantine equations by estimating linear forms in elliptic logarithms, Acta Arith. 67 (1994), 177-196.

[32] - - - On the elliptic logarithm method for elliptic diophantine equations: Reflections and an improvement, Experiment. Math. 8 (1999), 135-149.

[33] - - - Computing all integer solutions of a general elliptic equation, in: W. Bosma (ed.), ANTS-IV, Lecture Notes in Comput. Sci. 1838, Springer, Berlin, 2000, $551-561$.

[34] R. J. Stroeker and B. M. M. de Weger, On elliptic diophantine equations that defy Thue-The case of the Ochoa curve, Experiment. Math. 3 (1994), 1-12.

[35] N. Tzanakis, Explicit solution of a class of quartic Thue equations, Acta Arith. 64 (1993), 271-283.

[36] - Solving elliptic diophantine equations by estimating linear forms in elliptic logarithms. The case of quartic equations, ibid. 75 (1996), 165-190.

[37] M. Veluppillai, The equations $z^{2}-3 y^{2}=-2$ and $z^{2}-6 x^{2}=-5$, in: A Collection of Manuscripts Related to the Fibonacci Sequence, Fibonacci Assoc., Santa Clara, CA, 1980, 71-75.

[38] P. G. Walsh, On integer solutions to $x^{2}-d y^{2}=1, z^{2}-2 d y^{2}=1$, Acta Arith. 82 (1997), 69-76.

[39] - On two classes of simultaneous Pell equations with no solutions, Math. Comp. 68 (1999), 385-388.

[40] D. G. Zeng, More on simultaneous solutions of the Pell equations $x^{2}-2 y^{2}=1$ and $y^{2}-D z^{2}=4$, Math. Practice Theory 1995, no. 1, 81-83 (in Chinese).

Department of Mathematics

University of Crete

Iraklion, Greece

E-mail: tzanakis@math.uch.gr

http://www.math.uch.gr/selidamath/faculty/tzanakis.htm 